tively to systems of co-ordinates possessing arbitrary motion with respect to an inertial system. With the aid of these special fields we should be able to study the law which is satisfied in general by gravitational fields. In this connection we shall have to take account of the fact that the ponderable masses will be the determining factor in producing the field, or, according to the fundamental result of the special theory of relativity, the energy density-a magnitude having the transformational character of a tensor.

On the other hand, considerations based on the metrical results of the special theory of relativity led to the result that Euclidean metrics can no longer be valid with respect to accelerated systems of co-ordinates. Although it retarded the progress of the theory several years, this enormous difficulty was mitigated by our knowledgr that Euclidean metrics holds for small domains. As a consequence, the magnitude $d s$, which was physically defined in the special theory of relativity hitherto, retained its significance also in the general theory of relativity. But the co-ordinates themselves lost their direct significance, and degenerated simply into numbers with no physical meaning, the sole purpose of which was the numbering of the space-time points. Thus in the general theory of relativity the co-ordinates perform the same function as the Gaussian co-ordinates in the theory of surfaces. A necessary consequence of the preceding is that in such general co-ordinates the measurable magnitude $d s$ must be capable of representation in the form

$$
d s^{2}=\sum_{u v} g_{u v} d x_{u} d x_{v}
$$

where the symbols $g_{u v}$ are functions of the spacetime co-ordinates. From the above it also follows that the nature of the space-time variation of the factors $g_{u v}$ determines, on one hand the space- time metrics, and on the other the gravitational field which governs the mechanical behaviour of material points.

The law of the gravitational field is determined mainly by the following conditions : First, it shall be valid for an arbitrary choice of the system of co-ordinates; secondly, it shall be determined by the energy tensor of matter; and thirdly, it shall contain no higher differential coefficients of the factors $g_{u v}$ than the second, and must be linear in these. In this way a law was obtained which, although fundamentally different from Newton's law, corresponded so exactly to the latter in the deductions derivable from it that only very few criteria were to be found on which the theory could be decisively tested by experiment.

The following are some of the important questions which are awaiting solution at the present time. Are electrical and gravitational fields really so different in character that there is no formal unit to which they can be reduced? Do gravitational fields play a part in the constitution of matter, and is the continuum within the atomic nucleus to be regarded as appreciably nonEuclidean? A final question has reference to the cosmological problem. Is inertia to be traced to mutual action with distant masses? And connected with the latter: Is the spatial extent of the universe finite? It is here that my opinion differs from that of Eddington. With Mach, I feel that an affirmative answer is imperative, but for the time being nothing can be proved. Not until a dynamical investigation of the large systems of fixed stars has been performed from the point of view of the limits of validity of the Newtonian law of gravitation for immense regions of space will it perhaps be possible to obtain eventually an exact basis for the solution of this fascinating question.

\title{
Relativity: The Growth of an Idea.
}

\section{By E. Cunningham.}

$\mathrm{S}^{\mathrm{Ac}}$ CCHERI, in his "Logica Demonstrativa," published in 1697 , ten years after Newton's "Principia Mathematica," lays down a distinction between real and nominal definitions which should be kept in mind if we are to do justice to Newton. Euclid defines a square as a four-sided figure the sides of which are all equal, and the angles of which are all right-angles. That is what he means by the name "square." It is a nominal definition. It remains to be shown that such a figure exists. This is done in Book I., Prop. 46. The definition then becomes real. Euclid is not guilty of the error of presupposing the existence of the figure.

Newton prefixes to his principles of natural philosophy certain definitions of absolute, true, and mathematical space and time. The former remains fixed and immovable; the latter flows uniformly on, without regard to material bodies. He strives here against the imperfections of lanNo. 2677 , VOL. IO6] guage to give words to the thought in the back of his mind. The philosopher attacks him on these definitions; he has no right to presuppose that these words correspond to any reality. What then? Suppose these offending definitions removed, or recognised as purely nominal. Then the definitions of velocity, acceleration, mass, and force are nominal, too, and the whole of Newton's structure of dynamics is a paper scheme of words and relations which may or may not correspond to the world of sense.

But that is exactly what it is. That is what all scientific theory is, until experiment demonstrates that the correspondence exists. The justification of Newton's theory comes, not in the discovery of a time that flows uniformly on, but in the fact that the observed phenomena of the tides, of planetary motion, and of mechanics in general do fit on to his scheme. But the fit does not consist 
in the agreement of clock-time or solar time with absolute time. It is in the mutual agreement of the motions of clocks, earth, planets, moon, tides, and the rest; so that it matters not a whit whether the time variable used in the calculations flows uniformly on, or whether it is the merest variable number devoid of any physical significance whatever.

Thus the great paradox, the stumbling-block of absolute rotation, is not one of logic. Nature thrusts it upon us. While we would all admit that a priori rotation relative to empty space is a meaningless term, yet there remain Newton's bucket and Foucault's pendulum; and, strive as we will, Newton's way of looking at them remains the simplest. We would all fain think that "acceleration relative to empty space" is an empty phrase, and yet Nature is such that Newton's system of dynamics made modern science possible. Whatever we may say of Newton's definitions, therefore, his space and time are natural products of Nature's laboratory, to be purified, perhaps, but not to be rejected as spurious.

The nineteenth century was the æther age. From Arago, in I820, to Michelson and Morley (r88x-1905), followed by Trouton, Noble, Rankine, and Brace, vain attempts were made to. discover the earth's velocity through the æther. But what, after all, was this elusive medium? To Faraday doubtless it was as real as anything in the universe. His resolute insistence on the need for a medium of transmission of electrical action, his discovery of the induction of currents by changing magnetic fields, Maxwell's advocacy of his views, and the demonstration of the electromagnetic nature of light, led up directly to the electron theory of Lorentz and Larmor, in which the æther appears as the background of all material phenomena. Yet the ather, after all, was but a name, and the electron theory a formal scheme of relations; like Newton's scheme, it was to be verified by its correspondence with actual observation. When it comes to observation, the only possibility is to note the behaviour of material bodies. Thus the æther with the associated ideas of electric and magnetic intensity ranks pari passu with Newton's absolute time and space. It has no likeness with matter; it is even doubtful whether it is of such substantiality that any element of it has identity. It has been said of Larmor's account of it that it reduces the universe to a set of differential equations, which, of course, is to a great extent true of Newton's work also.

But the notable thing about this theory is that it leaves the problem of absolute motion and absolute rotation exactly at the point where Newton left it. It gives a reasonable account of the failure of Michelson and Morley and the rest to discover the earth's velocity relative to the æther, just as Newton's theory would show why its velocity in absolute space cannot be determined. The ætherbuilders succeeded too well, and constructed, not one, but an infinity of æthers, any one having a uniform translatory motion relative to any other.
But in the new theory, as in the old, a body may be said in an absolute sense to be devoid of rotational motion.

With the lack of determinateness in the æther goes a similar ambiguity in the measures of time and space. Each of these æthers has its proper scale of time and space. Events which are simultaneous in one æether are not simultaneous in another, and, since none can tell which is the true æther, none can tell whether two events are simultaneous or not. This is where the theories of Lorentz and Larmor lead us; and yet they only suggest it without demonstrating it. For neither of them professed to give a complete account of the structure of matter. The relations they propose for the rthereal action need to be supplemented by hypotheses as to the nature of the electron before they make a complete scheme; and when it comes to this Lorentz suggests a hypothesis, that of the contracting electron, which is devised specially for the purpose of getting the æther to conceal itself.

This brings us to the point at which Einstein makes his very modest suggestion (1905) of testing the hypothesis that in the nature of things we must not expect ever to find evidence of an absolute velocity of the earth through space. The ground had been well prepared. The electron theory as generally accepted was taken as it stood. The only new idea was that whereas his predecessors clung to the thought that one æther with its associated system of space-time measurement was real and the rest fictitious, Einstein, in the absence of any distinguishing features, ranks them all alike. To which some replied, "Give us back our absolute time and our æther." Yet Einstein had logic on his side, for the definitions of æther and of time are definitions quid nominis, not quid rei.

Looking back, we realise two great gaps in the special principle of relativity as Einstein propounded it in 1905. The first is the same which revealed itself in Newton's theory. "Uniform motion in a straight line" is left as a phrase with meaning, a situation as intolerable to the philosopher as the recognition of "absolute rest." Further, while it accepted tlie electromagnetic theory intact as a common basis of the structure of matter, it took no account of the other common property of matter, gravitation, of which so far the electromagnetic theory offered no explanation. Einstein at once realised the gaps, however, and saw a relation between them. Gravitational problems are problems of acceleration. The magnitude of the velocity of an observer does not affect his sense of gravity, but the experience of the man in the lift is that his acceleration does so. Thus Einstein perceived that to settle his difference with the philosopher he must first reconcile gravitation. Whether he would ever have done it without the genius of Minkowski we cannot tell. But as a matter of history the pure mathematician, who surely had learned much from Riemann and Helmholtz, was the first to emphasise the profound 
unity between the categories of space and time that was suggested by Einstein's work. A universe of determinate relations, in which everything proceeds according to known laws, is one which may be surveyed as a great whole in which past, present, and future are one. It is a fourdimensional unity, and for Einstein any plane section through it could be the present. The history of a particle is a simple chain of points in that whole, a "world-line."

Now events moved fast. Minkowski gave the notion of a four-dimension universe. Riemann had initiated a method of geometry in any number of dimensions; Einstein had glimpsed the possibility that, taking gravitation into account, light might be subject to acceleration, which, being interpreted in Minkowski's fashion, meant that the ordinary Euclidean expression for the element of length in a four-dimension space must be generalised. This made the work of Riemann not only useful, but also prophetic. In $r 853$ he had written that "the ground of the measure relations existing in the universe, if continuous, must consist in the binding forces acting upon it." This really implies the whole relativity doctrine; it asserts that the measure relations of the pheno- mena perceived in the universe are incapable of determination on any absolute scale, independent of the phenomena themselves. Sixty years later Einstein perceives that the gravitational field must be included among those binding forces, and must affect profoundly the measure relations in every physical aspect. Having foreseen this, Riemann had proceeded to develop a non-Euclidean geometry in any number of dimensions, and thus the germ of the calculus that. Einstein needed was created. With what success it was wielded is now well known.

Not so well known is the more recent work of Weyl. Einstein finds in universal gravitation the ground of the measure relations of the universe. But equally universal is the fact of electricity, and this universal "binding force" must equally take a part in those relations. The acuteness of Riemann's vision is doubly emphasised when we hear that Weyl discovers a further generalisation of his geometrical method which provides for electricity a place as natural and convincing as that taken by gravitation in Einstein's theory. But that is history yet in the making, and this article seeks only to traverse the course of history already complete.

\section{Relativity and the Eclipse Observations of May, I9I9.}

\section{By Sir Frank Dyson, F.R.S.}

I $\mathrm{N}$ I9I5 Prof. Einstein predicted, as a consequence of the generalised theory of relativity, that a ray of light from a star would be bent in its passage through the sun's gravitational field. The amount of this deflection he gave as $\mathrm{I} \cdot 75^{\prime \prime}(a / r)$, where $a$ is the sun's radius, and $r$ the nearest distance of the ray to the sun's centre. As a ray of light reaches us in the direction of the tangent to its path, the apparent position of a star, photographed during an eclipse of the sun, should therefore be displaced by an angle $1 \cdot 75^{\prime \prime}(a / r)$ outwards from the sun's centre. The field of stars surrounding the sun should thus be distorted in a definite manner by an amount within the range of accurate astronomical observation. In Fig. I, if $a, b, c$ are the positions of the stars as seen at ordinary times, and if $\mathrm{S}$ is the centre of the sun during an eclipse, then the positions in which the stars are seen or photographed during an eclipse will be $\mathrm{A}, \mathrm{B}, \mathrm{C}$. If $a$ were at a distance $3 \mathrm{O}^{\prime}$ from the sun's centre the displacement $a \mathrm{~A}$ would be $0.87^{\prime \prime}$, and if B were at a distance $9 \mathrm{o}^{\prime}$ from the sun's centre its displacement would be $0 \cdot 27^{\prime \prime}$. These are, roughly speaking, the greatest and least displacements which can be obtained in practice. Nearer than $30^{\prime}$ from the sun's centre a star's image is liable to be drowned in the corona. At greater distances than $90^{\prime}$ good images can scarcely be obtained with a simple object glass, while a doublet or other combination introduces some difficulties.

These displacements, though small, are ten times as large as those met with in determinaNo. 2677 , VOL. IO6] tions of stellar parallax, and are determined in a precisely similar manner by comparison of photographs taken at different epochs. The field of stars photographed during the eclipse must be compared with the same field photographed when the sun is in another part of the sky. Experience shows that telescopes of as long focal length as practicable should be used, and that the eclipse field and comparison field should be phorographed under as nearly as possible identical con-

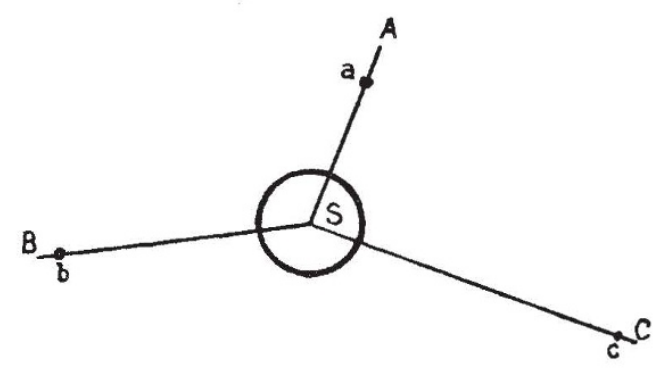

FIG. 1.

ditions as regards both the instruments themselves and the position of the field in the sky. With these precautions there was every reason to suppose that, given favourable weather, successful results would be obtained.

In the short time available for preparation it was not possible to arrange for the telescopes to be mounted equatorially, and the field of stars was reflected into fixed telescopes by cœlostat 